\title{
Use of Contrast-Enhanced Ultrasound for Detecting the Disease Activity of the Carotid Artery in Takayasu Arteritis
}

\author{
Chaolun Li, MD ${ }^{a, *}$, Lingying Ma, MD ${ }^{b}$, Linjin Huang, MD ${ }^{c}$, Hong Han, MD ${ }^{a}$, Lingdi Jiang, MD ${ }^{b}$, \\ Wenping Wang, $M^{a}$ \\ ${ }^{a}$ Department of Ultrasound, Zhongshan Hospital, Fudan University, Shanghai, China; ${ }^{b}$ Department of Rheumatology, Zhongshan \\ Hospital, Fudan University, Shanghai, China, ${ }^{c}$ Department of Ultrasound, Xiamen Branch, Zhongshan Hospital, Fudan University, \\ Xiamen, China
}

Received February 23, 2020; revision received March 16, 2020; accepted March 22, 2020.

Objective: To investigate whether contrast-enhanced ultrasound (CEUS) is helpful for assessing the disease activity of Takayasu arteritis (TA).

\begin{abstract}
Methods: Eighty-four patients with TA were examined with ultrasound (US) and CEUS. Intima-media thickness (IMT) of both sides of the common carotid artery was measured for each patient. Semiquantitative analyses of contrast enhancement within the arterial wall were performed with a visual interpretation scale. Disease activity was determined by one experienced physician based on Physician Global Assessment criteria.

Results: Carotid CEUS revealed that 6 arteries showed no enhancement $(7.1 \%, 6 / 84)$ while 78 arteries showed homogeneous enhancement within the thickened wall $(92.9 \%, 78 / 84)$. Grade 1 enhancement was noted in 40 patients, and grade 2 in 38 patients. IMT of the active group was significantly thicker than that of the inactive group $(2.4 \pm 0.9 \mathrm{~mm}$ vs. $1.8 \pm 0.5 \mathrm{~mm}$, $P=0.001)$ on B-mode US, and slightly correlated with ESR $(r=0.344, P<0.05)$ and CRP $(r=0.261, P<0.05)$. Grade 2 enhancement was observed more in the active patients $(61.7 \%$ vs. $41.3 \%, P=0.001)$ on CEUS.
\end{abstract}

Conclusions: CEUS is a convenient and non-invasive imaging modality that is useful for evaluating disease activity in TA patients by assessing the vascularization within the carotid wall.

Key words: Takayasu arteritis; Contrast-enhanced; Ultrasound; Carotid artery

Advanced Ultrasound in Diagnosis Contrast and Therapy 2020;03:189-194

DOI: $10.37015 / A U D T .2020 .200010$

$\mathrm{T}$ akayasu arteritis (TA) is a rare chronic vasculitis affecting large vessels such as the aorta and its main branches. It is characterized by granulomatous inflammation of the vessel wall. Initial inflammatory changes are associated with vasa vasoritis and proliferation of the vaso vasorum, and the vessels represent the portal of entry of inflammatory cells to the artery wall [1]. This inflammation leads to wall thickening, stenosis, thrombus formation, and ultimately, organ ischemia [2]

Patients with TA present with non-specific symptoms such as anterior cervicalgia, fever, fatigue, headaches, and weight loss. Diagnosis is mainly based on clinical and angiographic manifestations. Management of TA is challenging because of the lack of specific clinical, laboratory, and imaging findings reflecting disease activity, especially arterial wall inflammation [3]. Acute phase reactants (APR), including serum $\mathrm{C}$ reactive

\footnotetext{
* Corresponding Author: Department of Ultrasound, Zhongshan Hospital, Fudan University, 180 Fenglin Road, Shanghai, China e-mail: li.chaolun@zs-hospital.sh.cn
} 
protein (CRP) and erythrocyte sedimentation rate (ESR), are commonly used, but they do not always correlate with disease activity [4]. Imaging methods, including color Doppler ultrasound (US), computerized tomographic angiography (CTA), magnetic resonance angiography (MRA), and positron emission tomography (PET), are useful in diagnosing TA in the early, pre-stenotic phase, however their role in monitoring disease activity remains unclear so far [4]. Moreover, exposure to large amount of radiation and iodinated contrast limits the use of CTA and PET as routine follow-up imaging modalities.

Contrast-enhanced ultrasound (CEUS) has been increasingly used to evaluate carotid artery abnormalities. Studies have demonstrated that CEUS, a convenient and non-invasive imaging method, could be used to assess neovascularization and vaso vasorum in carotid atherosclerotic plaques [5, 6]. More recently, carotid CEUS has been proposed to be helpful for the evaluation of the disease activity of carotid artery in TA by assessing the vascularization in the artery walls in small research populations [7-10]. In this study, we evaluated the degree of CEUS enhancement within the carotid artery wall with visual scale, and we correlated these results with clinical findings and APR to investigate whether CEUS is helpful for assessing the disease activity of TA.

\section{Materials and Methods}

\section{Patients}

This study was approved by our institutional review board (Application ID: TA2016-168), and informed consent was obtained from all patients before the examination. From February 2016 to September 2017, 84 patients (11 men, 73 women; age range, 19-57 years; mean age, $31.9 \pm 10.0$ years) were enrolled in this study. All the patients were clinically diagnosed as TA based on The American College of Rheumatology (ACR) criteria [11]. Either bilateral or unilateral carotid arteries were confirmed in all 84 patients by imaging examinations (US, CTA, or MRA). Patients with a history of heart failure, myocardial infarction, or unstable angina were excluded from this study, as these conditions contradict the use of ultrasound contrast agent (UCA). Three patients with occluded common carotid arteries on both sides were also excluded from this study.

\section{Standard US and CEUS examinations}

Patients underwent CEUS after routine US examination. The both US examinations were performed with a Philips Elite scanner (Philips Healthcare, Bothell, USA) equipped with a linear-array L9-3 MHz probe and pulse inversion harmonic imaging software. All conventional US and CEUS examinations were performed by two experienced radiologists. When CEUS was performed, the mechanical index was set at 0.07 , and the gain at $90 \%$. The focal zone was positioned at a depth of 2-3 cm depending on the depth of the carotid artery. Time gain compensation was adjusted to obtain a homogeneous image of the carotid artery while reducing the noise from the arterial wall. These values were preset for the CEUS examination in all patients.

With grayscale US, the right and left common carotid arteries (CCA) along with internal and external carotid arteries were examined in longitudinal and transverse projections. The thickness of carotid wall was measured on both sides of the far wall of the CCA at the thickest spot on a frozen frame of a suitable magnified image, and the mean value was obtained from 3 measurements for each side. Color Doppler US was performed to detect the blood flow. Then, peak systolic velocity (PSV) was measured with spectral Doppler using an insonation angle of less than 60 degrees.

CEUS was performed in longitudinal projections of the CCA with the thicker carotid wall. If the carotid artery was occluded on one side, CEUS was performed on the other side. SonoVue (Bracco, Milan, Italy), a suspension of phospholipid-stabilized sulfur hexafluoride (SF6) microbubbles, was used as the contrast agent. For each injection, the patient was given a bolus dose of $2 \mathrm{~mL}$ UCA through a peripheral intravenous line, followed by a $5 \mathrm{~mL}$ normal saline flush. The probe was held steady over the target lesion for 1 minute during the CEUS examination. A real-time US imaging cine-loop was digitally stored for further analysis.

\section{CEUS image interpretation}

On CEUS images, the presence of dynamic movement of the echogenic reflectors (UCA bubbles) within the carotid lesions was defined as evidence of enhancement, whereas fixed echogenic signals were considered to be tissue acoustic reflector [6].

Semiquantitative analyses were performed off-line by one experienced radiologist who were unaware of the clinical data. The degree of contrast enhancement in each carotid lesions was categorized on a visual scale as follows $[9,12,13]$ : grade 0 , no enhancement, indicating no appearance of microbubble contrast agent in the thickened arterial wall; grade 1, limited or moderate enhancement, indicating limited to moderate visible appearance of moving microbubbles in the thickened arterial wall; or grade 2, severe enhancement, indicating extensive visible appearance of microbubbles (Fig. 1).

\section{Laboratory tests}

ESR and CRP levels were measured at the time of CEUS examinations. The upper limit of the normal 
reference range of ESR was $<30 \mathrm{~mm} / \mathrm{H}$ and of CRP was $3 \mathrm{mg} / \mathrm{L}$.

\section{Clinical assessment of disease activity}

TA disease activity was determined by one experienced physician based on Physician Global Assessment (PGA) criteria.[14] General activity is defined broadly with the presence of categorical definitions: (1) systemic features, such as fever, musculoskeletal (no other cause identified); (2) elevated ESR; (3) features of vascular ischemia or inflammation, such as claudication, diminished or absent pulse, bruit, vascular pain (carotodynia), asymmetric blood pressure in either upper or lower limbs (or both); (4) typical angiographic features.
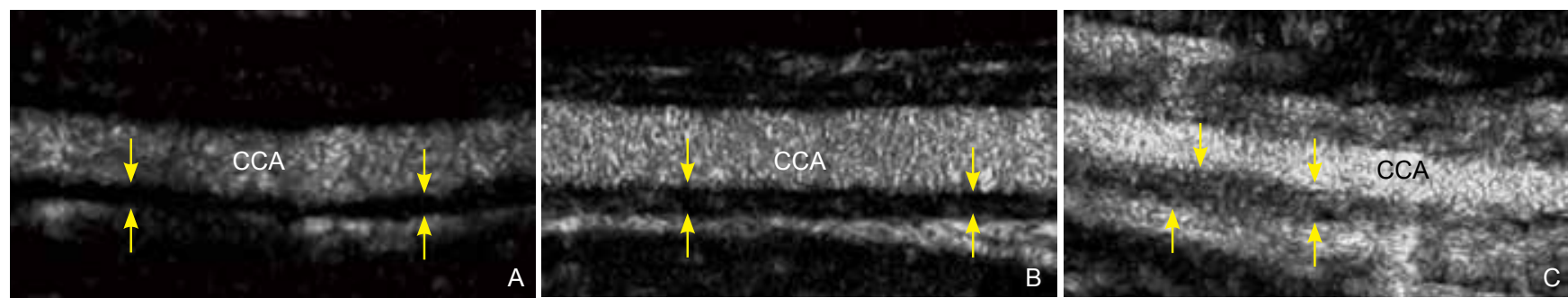

Figure 1 Degree of CEUS enhancement of the carotid lesions. (A) Grade 0, no enhancement, indicating no appearance of microbubble contrast agent in the thickened arterial wall; (B) Grade 1, limited or moderate enhancement, indicating limited to moderate visible appearance of moving microbubbles in the thickened arterial wall; (C) Grade 2, severe enhancement, indicating extensive visible appearance of microbubbles. CCA, common carotid artery.
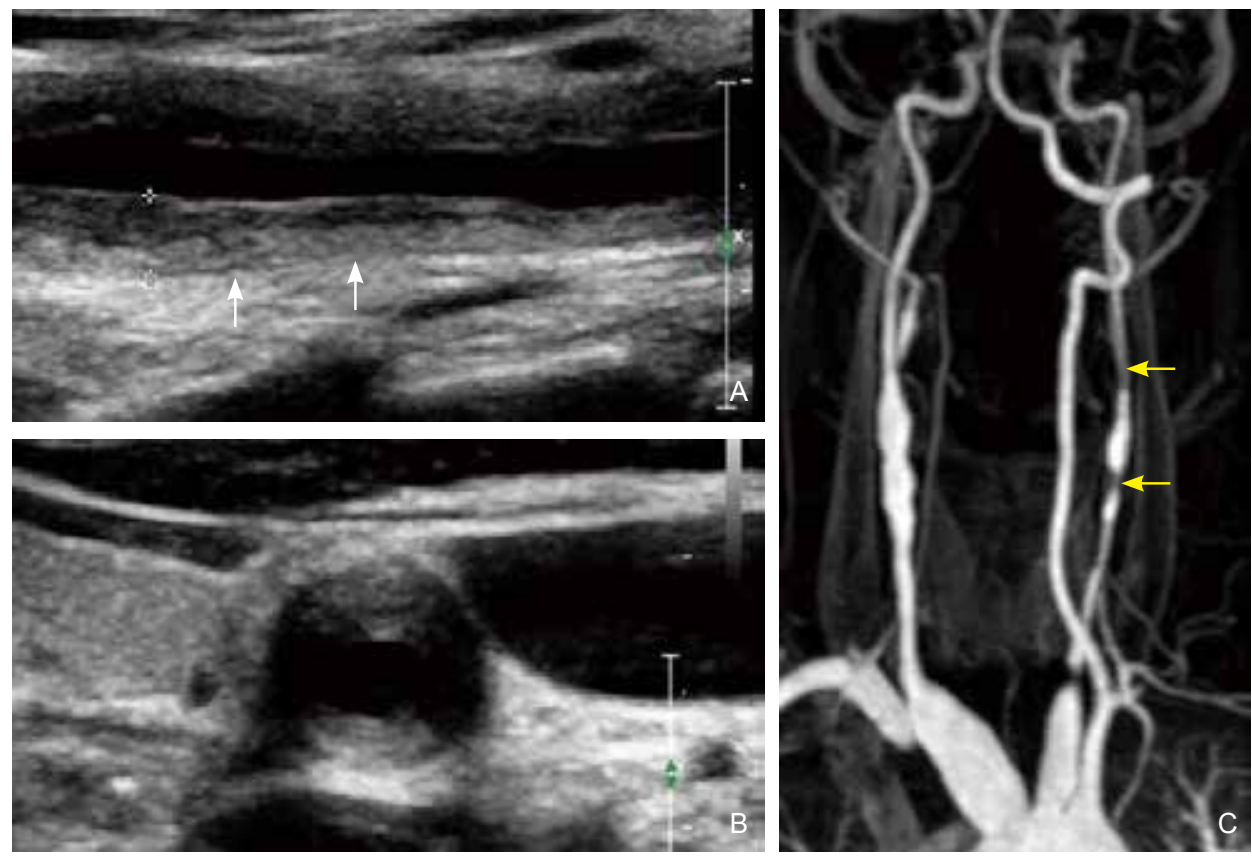

Figure 2 Carotid B-mode US in a 31-year-old woman with TA. Longitudinal (A) and transversal (B) images demonstrated the concentric thickening of the left common carotid arterial wall with narrowed irregular lumen. The boundary between the adventitia and the surrounding soft tissue was blurred (arrows). (C) Contrast-enhanced MRA showed steno-occlusive left common carotid artery (arrows).

\section{Statistical analysis}

Data were analyzed with SPSS version 22.0. Continuous variables were presented as mean \pm standard deviation (SD); medians were used for nonnormally distributed data. Discrete data were presented as frequencies. Numeric variables were compared with independent t-test. Comparison of the categorical variables was performed with chi-square test. A $P$ value $<.05$ indicated statistical significance.

\section{Results}

\section{Clinical data}

Out of the 84 patients, 47 patients were classified at the time the CEUS examination as having active TA based on PGA criteria, and the other 37 as inactive. In all, 35 (41.7\%) had not been treated for TA before the examination. The other 49 patients $(58.3 \%)$ had been treated with corticosteroids and immunosuppressive 
therapy, and had disease duration ranging from 1 month to 27 years (median: 29 months) from the onset of symptoms.

\section{Conventional US findings}

All patients showed the presence of long, smooth concentric thickening of the carotid arterial wall on B-mode US (Fig. 2). Bilateral vascular lesions were noted in 67 patients $(79.8 \%)$, and unilateral lesions in 17 patients $(20.2 \%)$. The whole CCA was involved in 72 patients $(85.7 \%)$, while only the proximal CCA was involved in the remaining 12 patients $(14.3 \%)$. All of the vascular lesions presented as hypoechoic or isoechoic on B-mode US (Fig. 2). The boundary between the adventitia and the surrounding soft tissue was blurred. The mean thickness of the arterial wall was $2.1 \pm 0.8 \mathrm{~mm}$ (range $1.2-5.6 \mathrm{~mm}$ ). Mean PSV was $1.37 \pm 0.99 \mathrm{~m} / \mathrm{s}$ (range 0.17-3.95 m/s). The arterial lumen could be narrowed or irregular. Ten CCA were noted as being occluded on US: 7 on the right side, and 3 on the left side.

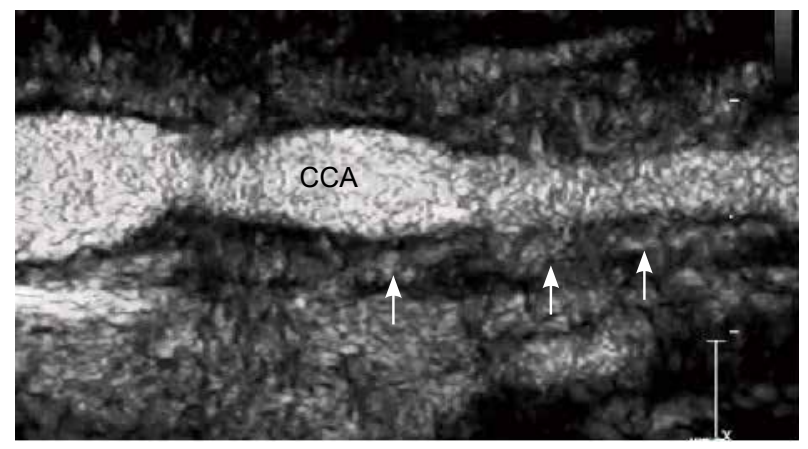

Figure 3 Same patient in Figure 2. Longitudinal CEUS image of the left CCA. The contrast agent improved the visualization of the border of the vascular lesion, and CEUS showed homogeneous enhancement within the thickened wall, indicating wall vascularization (arrows). CCA, common carotid artery.

\section{CEUS findings}

Carotid CEUS demonstrated homogeneous enhancement with certain degree within the thickened carotid wall (Fig. 3 ) in 78 arteries $(92.9 \%, 78 / 84)$. Only 6 arteries $(7.1 \%, 6 / 84)$ showed no enhancement on CEUS. Grade 1 enhancement was noted in 40 patients, and grade 2 in 38 patients (Table 1).

Table 1 Degree of CEUS enhancement showed in active and inactive TA patients

\begin{tabular}{cccc}
\hline Item & Active (\%) & Inactive (\%) & Total \\
\hline Grade 0 & 0 & $6(16.2 \%)$ & 6 \\
Grade 1 & $18(38.3 \%)$ & $22(59.5 \%)$ & 40 \\
Grade 2 & $29(62.7 \%)$ & $9(24.3 \%)$ & 38 \\
\hline Total & 47 & 37 & 84 \\
\hline
\end{tabular}

\section{Comparison between the active and inactive groups}

ESR levels were significantly higher in the active group compared to the inactive group $(50.72 \pm 34.55 \mathrm{~mm} / \mathrm{H}$ vs. $20.53 \pm 21.25 \mathrm{~mm} / \mathrm{H}, P<0.001)$ and $\mathrm{CRP}(41.37 \pm$ $53.85 \mathrm{mg} / \mathrm{L}$ vs. $8.53 \pm 11.66 \mathrm{mg} / \mathrm{L}, P=0.001)$.

The thickness of the affected carotid wall was significantly thicker of the active group compared to the inactive group $(2.4 \pm 0.9 \mathrm{~mm}$ vs. $1.8 \pm 0.5 \mathrm{~mm}, P=0.001)$ on B-mode US. It was slightly correlated with ESR ( $r=$ $0.344, P<0.05)$ and $\operatorname{CRP}(r=0.261, P<0.05)$.

Patients with clinically active disease showed more enhancement on CEUS. In particular, Grade 2 enhancement on CEUS was observed more frequently in the active patients $(61.7 \%$ vs. $41.3 \%, P=0.001)$ (Table 1). The 6 patients who showed no enhancement were all clinically defined as inactive. In patients with different enhancement intensity, median ESR $(\mathrm{mm} / \mathrm{H})$ was $4.5,20.5$, and $41.0(P<0.05)$, respectively; and median CRP $(\mathrm{mg} / \mathrm{L})$ was $0.35,0.70$, and $19.00(P<0.05)$, respectively.

All patients were divided into either the normal group or the abnormal group according their ESR and CRP levels. Either the thickness of carotid wall or PSV showed significant difference between the 2 groups. More patients in the abnormal group showed grade 2 enhancement on CEUS (Table 2).

Table 2 Comparison of US and CEUS parameters in patients with different ESR and CRP level

\begin{tabular}{|c|c|c|c|c|c|c|}
\hline \multirow{2}{*}{ Item } & \multicolumn{3}{|c|}{ Erythrocyte sedimentation rate (ESR) } & \multicolumn{3}{|c|}{$\mathrm{C}$ reactive protein (CRP) } \\
\hline & Normal & Abnormal & $P$ value & Normal & Abnormal & $P$ value \\
\hline Number & 44 & 40 & & 28 & 56 & \\
\hline Intima-media thickness (mm) & $2.0 \pm 0.8$ & $2.3 \pm 0.8$ & 0.110 & $2.0 \pm 0.8$ & $2.2 \pm 0.8$ & 0.29 \\
\hline Peak systolic velocity (m/s) & $1.25 \pm 1.00$ & $1.48 \pm 0.99$ & 0.323 & $1.32 \pm 0.94$ & $1.37 \pm 1.02$ & 0.835 \\
\hline Grade 2 enhancement (\%) & $15(34.1 \%)$ & $23(57.5 \%)$ & 0.025 & $6(21.4 \%)$ & $32(51.7 \%)$ & 0.001 \\
\hline
\end{tabular}




\section{Discussion}

Carotid artery abnormalities occur in $45 \%$ to $84 \%$ patients with TA [15]. Conventional US has long been used to evaluate such carotid abnormalities since it assesses arterial wall abnormalities with sensitivity that is up to 10 -fold greater than MRI and displays a resolution of $0.1-0.2 \mathrm{~mm}$ [16]. Results in this study showed that in the group of patients with active TA, the thickness of the carotid wall as measured by conventional US was significantly greater than that of the inactive group $(2.4 \pm 0.9 \mathrm{~mm}$ vs. $1.8 \pm 0.5 \mathrm{~mm}, P=0.001)$. Our US findings were consistent with a previous study that reported US may also be useful in determining disease activity by demonstrating hypoechogenicity and mural thickening in active lesions [16-18]. However, Giordana et al. recently reported on a 35-year-old woman with TA in which CEUS showed marked diminution of the arterial wall enhancement and vasa vasorum opacification after treatment but no change in the thickness of arterial wall during the same period [8].

CEUS has been reported not only to improve the visualization of the carotid lumen border but also to allow the assessment of the neovascularization in carotid atherosclerotic plaques [5,19]. The degree of intraplaque neovascularization on CEUS has been found to correlate highly with the inflammation evaluated with histopathologic analysis of the plaque after endarterectomy $[12,20,21]$. Histopathological findings of TA have revealed that the arterial wall contains neovessels in the deep intima associated with the adventitial vasa vasorum, which could be a marker of the disease activity [1]. Recently, researchers proposed that CEUS might be useful to evaluate disease activity and treatment response by assessing vascularization in the affected arterial wall [7-10].

In this study, all 6 patients who presented with no enhancement on CEUS were clinically classified as inactive according to PGA criteria. Meanwhile more of the patients with grade 2 enhancement on CEUS were in the active group than in the inactive group. Our finding that clinically active TA patients showed more enhancement on CEUS is consistent with previously published results $[9,13]$. Germanò et al. [13] used both CEUS and PET to investigate 31 patients with large vessel vasculitis (14 with TA, 17 with giant cell arteritis) and revealed that the carotid CEUS vascularization grade significantly correlated with the grade of vascular inflammation on PET. Their results confirmed the value of CEUS for evaluating the disease activity of TA. However, in our study 18 active patients and 22 inactive patients showed grade 1 enhancement on carotid CEUS in this study. This result suggested that CEUS results should be integrated with clinical findings to evaluate the disease activity in individual patients with TA, and further studies and more objective standards are needed.

Acute inflammation reactants, including ESR and CRP have been widely used to assess disease activity and response to treatment. However, inflammatory markers do not reliably correlate with disease activity. In a study from Mayo Clinic, inflammatory markers were elevated at diagnosis in only 85 of 119 patients (71\%) [22]. In patients with apparent clinical and laboratory remission, arterial specimens may show histological signs of vasculitis [23]. Herlin et al. [10] reported that a 43-year-old patient who was under treatment for TA experienced an ischaemic stroke with normal biological inflammatory parameters. However, CEUS demonstrated progressive arterial wall enhancement in this patient. Given the ischaemic event and CEUS findings, the dose of corticosteroids was increased despite the normal biological parameters. Three months later, follow-up CEUS showed no artery wall enhancement in the same patient, while the B-mode ultrasound appearance did not change. In this study involving 47 clinically active patients, 15 (31.9\%) showed normal ESR and CRP level. Meanwhile, all active patients showed enhancement on CEUS, and 29 (61.7\%) showed grade 2 enhancement. These results suggested that CEUS might be more sensitive than ESR and CRP for evaluating disease activity.

Our study had several limitations. First, the degree of the enhancement on CEUS was visually determined, and we used no objective standardization of CEUS for the evaluation of carotid wall vascularization. In addition, the disease activity was determined by physicians using PGA criteria, which are based on clinical and laboratory data, because it is difficult to get the histological data of the carotid wall abnormalities in the TA patients. Moreover, CEUS findings of the response to treatment were not included in this study and require further investigation.

In conclusion, CEUS is a convenient and noninvasive imaging modality that shows promise for evaluating disease activity in patients with TA by assessing the vascularization within the carotid wall.

\section{Conflict of Interest}

The authors have no conflict of interest to declare.

\section{References}

[1] Vaideeswar P, Deshpande JR. Pathology of Takayasu arteritis: A brief review. Ann Pediatr Cardiol 2013; 6: 52-8.

[2] Johnston SL, Lock RJ, Gompels MM. Takayasu arteritis: a review. $J$ Clin Pathol 2002; 55: 481-6.

[3] Keser G, Direskeneli H, Aksu K. Management of Takayasu arteritis: a systematic review. Rheumatology (Oxford) 2014; 53: 793-801. 
[4] Direskeneli H, Aydin SZ, Merkel PA. Assessment of disease activity and progression in Takayasu's arteritis. Clin Exp Rheumatol 2011; 29: S86-S91.

[5] Feinstein SB. Contrast ultrasound imaging of the carotid artery vasa vasorum and atherosclerotic plaque neovascularization. $\mathrm{J} \mathrm{Am} \mathrm{Coll}$ Cardiol 2006; 48: 236-43.

[6] Coli S, Magnoni M, Sangiorgi G, Marrocco-Trischitta MM, Melisurgo G, Mauriello A, et al. Contrast-enhanced ultrasound imaging of intraplaque neovascularization in carotid arteries: correlation with histology and plaque echogenicity. $\mathrm{J} \mathrm{Am} \mathrm{Coll}$ Cardiol 2008; 52: 223-30.

[7] Magnoni M, Dagna L, Coli S, Cianflone D, Sabbadini MG, Maseri A. Assessment of Takayasu arteritis activity by carotid contrastenhanced ultrasound. Circ Cardiovasc Imaging 2011; 4: e1-e2.

[8] Giordana P, Baque-Juston MC, Jeandel PY, Mondot L, Hirlemann J, Padovani B, et al. Contrast-enhanced ultrasound of carotid artery wall in Takayasu disease: first evidence of application in diagnosis and monitoring of response to treatment. Circulation 2011; 124: 245 7.

[9] Schinkel AF, van den Oord SC, van der Steen AF, van Laar JA, Sijbrands EJ. Utility of contrast-enhanced ultrasound for the assessment of the carotid artery wall in patients with Takayasu or giant cell arteritis. Eur Heart J Cardiovasc Imaging 2014; 15: 541-6.

[10] Herlin B, Baud JM, Chadenat ML, Pico F. Contrast-enhanced ultrasonography in Takayasu arteritis: watching and monitoring the arterial inflammation. BMJ case reports. DOI:10.1136/bcr-2015211094.

[11] Arend WP, Michel BA, Bloch DA, Hunder GG, Calabrese LH, Edworthy SM, et al. The American College of Rheumatology 1990 criteria for the classification of Takayasu arteritis. Arthritis Rheum 1990; 33: 1129-34.

[12] Staub D, Partovi S, Schinkel AF, Coll B, Uthoff H, Aschwanden M, et al. Correlation of carotid artery atherosclerotic lesion echogenicity and severity at standard US with intraplaque neovascularization detected at contrast-enhanced US. Radiology 2011; 258: 618-26.

[13] Germano G, Macchioni P, Possemato N, Boiardi L, Nicolini A, Casali M, et al. Contrast-Enhanced Ultrasound of the Carotid Artery in Patients with Large Vessel Vasculitis: Correlation With Positron Emission Tomography Findings. Arthritis Care Res (Hoboken) 2017; 69: 143-9.

[14] Kerr GS, Hallahan CW, Giordano J, Leavitt RY, Fauci AS, Rottem M, et al. Takayasu arteritis. Ann Intern Med 1994; 120: 919-29.

[15] Park SH, Chung JW, Lee JW, Han MH, Park JH. Carotid artery involvement in Takayasu's arteritis: evaluation of the activity by ultrasonography. J Ultrasound Med 2001; 20: 371-8.

[16] Romera-Villegas A, Vila-Coll R, Poca-Dias V, Cairols-Castellote MA. The role of color duplex sonography in the diagnosis of giant cell arteritis. J Ultrasound Med 2004; 23: 1493-8.

[17] Seth S, Goyal NK, Jagia P, Gulati G, Karthikeyan G, Sharma S, et al. Carotid intima-medial thickness as a marker of disease activity in Takayasu's arteritis. Int J Cardiol 2006; 108: 385-90.

[18] Wang Y, Li JC, Liu H, Wang L, Qi ZH, Kong J. Ultrasound features in the diagnosis of Takayasu' $\mathrm{s}$ arteritis with carotid artery involvement and evaluation of disease activity. Med J PUMCH 2014; 5: $81-7$.

[19] Kono Y, Pinnell SP, Sirlin CB, Sparks SR, Georgy B, Wong W, et al. Carotid arteries: contrast-enhanced US angiography--preliminary clinical experience. Radiology 2004; 230: 561-8.

[20] Hoogi A, Adam D, Hoffman A, Kerner H, Reisner S, Gaitini D. Carotid plaque vulnerability: quantification of neovascularization on contrast-enhanced ultrasound with histopathologic correlation. Am J Roentgenol 2011; 196: 431-6.

[21] Li C, He W, Guo D, Chen L, Jin X, Wang W, et al. Quantification of carotid plaque neovascularization using contrast-enhanced ultrasound with histopathologic validation. Ultrasound Med Biol 2014; 40 1827-33.

[22] Schmidt J, Kermani TA, Bacani AK, Crowson CS, Cooper LT, Matteson EL, et al. Diagnostic features, treatment, and outcomes of Takayasu arteritis in a US cohort of 126 patients. Mayo Clin Proc 2013; 88: 822-30.

[23] Mavrogeni S, Dimitroulas T, Chatziioannou SN, Kitas G. The role of multimodality imaging in the evaluation of Takayasu arteritis. Semin Arthritis Rheum 2013; 42: 401-12. 